\title{
Spin polarization dynamics in the Bjorken-expanding resistive MHD background
}

\author{
Rajeev Singh $\oplus^{1, *}$ Masoud Shokri® ${ }^{2, \dagger}$ and Radoslaw Ryblewski® ${ }^{1, \$}$ \\ ${ }^{1}$ Institute of Nuclear Physics Polish Academy of Sciences, PL-31-342 Kraków, Poland \\ ${ }^{2}$ IPM, School of Particles and Accelerators, P.O. Box 19395-5531, Tehran, Iran
}

(Received 8 March 2021; accepted 3 May 2021; published 26 May 2021)

\begin{abstract}
Evolution of spin polarization in the presence of external electric field is studied for collision energies $\sqrt{s_{\mathrm{NN}}}=27 \mathrm{GeV}$ and $\sqrt{s_{\mathrm{NN}}}=200 \mathrm{GeV}$. The numerical analysis is done in the perfect-fluid Bjorkenexpanding resistive magnetohydrodynamic background and novel results are reported. In particular, we show that the electric field plays a significant role in the competition between expansion and dissipation.
\end{abstract}

DOI: 10.1103/PhysRevD.103.094034

\section{INTRODUCTION}

In recent years, relativistic hydrodynamics has become a commonly accepted tool for the description of relativistic heavy-ion collisions [1-5], which allows us to draw a uniform picture of the complicated processes taking place in these events. It has been quite successful in describing the collective phenomena [6], and, hence, it is supposed to be applicable to explain the QGP (quark-gluon-plasma) dynamics from the very early stages of evolution $[7,8]$. Despite the triumph of hydrodynamics in explaining physical observables, there are certain quantum aspects of the produced QCD matter that may not be understood using the standard formulations of relativistic hydrodynamics and indicate directions for many new investigations. In particular, recent measurements of spin polarization of $\Lambda$ hyperons [9-13] indicate that the incorporation of spin degrees of freedom into the standard hydrodynamics framework may be necessary for understanding the spin polarization of final hadrons. The first attempt to formulate relativistic hydrodynamics with spin as a dynamic quantity has been proposed in Ref. [14]; for various follow-up theoretical investigations see, for instance [15-29]. Other theoretical studies [30-49] deal mainly with the spin polarization during the freeze-out stage of heavy-ion collisions, where they consider that the thermal vorticity is the basic hydrodynamic quantity which gives rise to spin polarization.

\footnotetext{
*rajeev.singh@ifj.edu.pl

†mshokri@ipm.ir

†radoslaw.ryblewski@ifj.edu.pl
}

Published by the American Physical Society under the terms of the Creative Commons Attribution 4.0 International license. Further distribution of this work must maintain attribution to the author(s) and the published article's title, journal citation, and DOI. Funded by SCOAP .
Grounded on basic physical arguments [50] and simulations [51,52], large electromagnetic (EM) fields are produced during heavy-ion collisions. The typical scales of the initial field strength are of the order of $e E / m_{\pi}^{2} \sim$ $e B / m_{\pi}^{2} \sim \mathcal{O}(1)$, with $m_{\pi}$ being the pion mass and $e$ denoting the elementary electric charge. If the EM fields do not decay too quickly, then they may modify different aspects of the fireball dynamics including the dynamics of spin polarization. Although the production of large EM fields in heavy-ion collisions is not of any doubt, their dynamics is not yet settled. Thus, recent years have observed a significant attraction to the applications of both analytical [53-58] and numerical [59,60] solutions of relativistic magnetohydrodynamics (MHD) to heavy-ion collisions.

In this work, we assume the fluid in the microscopic scale to be composed of noninteracting quarklike quasiparticles of $N_{f}$ flavors in equilibrium, which admits a kinetic description according to the Boltzmann-Vlasov (BV) equation. By this virtue, we are not taking into account the direct coupling between the EM fields and spin degrees of freedom. The stationary solution of the BV equation is obtained, in particular, in Ref. [61] as the zeroth order in $\hbar$ expansion. In this solution, modification of the chemical potential permits the electric field to exist in equilibrium [62]. Although the electric field vectors may cancel out in the event-by-event averaging [51], the modifications of thermodynamics that they induce, do not. We use the stationary solution to the BVequation to derive the modification of hydrodynamic variables in equilibrium. The results are then plugged into the MHD equations, with the solutions of the Maxwell equations in the case of Bjorken flow derived in Ref. [55], to find the dynamics of temperature and chemical potential. We finally use the acquired background dynamics in the spin conservation law to study the spin polarization dynamics. The evolution of the hydrodynamic variables is modified both 
by the Joule heating $(\mathrm{JH})$ term at the macroscopic level, and the modification of the thermodynamics at the microscopic level. Consequently, the competition between the JH and expansion gives rise to novel results.

The structure of the manuscript is as follows: we start by modifying the perfect-fluid background in the presence of the external electric field in Sec. II and setting up the necessary hydrodynamic framework for the study of spin polarization. In Sec. III the details about the spin polarization tensor and the form of the spin tensor are given. Sec. IV deals with the evolution of EM fields and conservation laws. In Sec. V we present numerical results for the thermodynamic variables and spin polarization coefficients in the perfect-fluid background. Finally, we summarize the key results and interpretations of our work and outline possible future extensions in Sec. VI.

Notation and conventions. - In this paper, we use "mostly minus" metric convention. The scalar (or dot) product of two four vectors $a^{\alpha}$ and $b^{\alpha}$ reads $a \cdot b=$ $a^{\alpha} b_{\alpha}=g_{\alpha \beta} a^{\alpha} b^{\beta}=a^{0} b^{0}-\boldsymbol{a} \cdot \boldsymbol{b}$, where three vectors are denoted by bold font. For the Levi-Civita tensor $\epsilon^{\alpha \beta \gamma \delta}$ we adopt the convention $\epsilon^{0123}=-\epsilon_{0123}=+1$. We denote the Lie derivative of a tensor $X$ of arbitrary rank with respect to a vector $V$ as $£_{V} X$. We use a shorthand notation for antisymmetrization by a pair of square brackets. For example, for arbitrary rank-two covariant tensor $M$ we have $M_{[\mu \nu]}=1 / 2\left(M_{\mu \nu}-M_{\nu \mu}\right)$. Throughout the paper we use natural units, i.e., $c=\hbar=k_{B}=1$.

\section{HYDRODYNAMIC EQUATIONS IN THE PRESENCE OF ELECTROMAGNETIC FIELDS}

The EM fields may modify the spin hydrodynamics formalism in different ways. In the present work, we study the dynamics of spin polarization in the presence of a background gauge field $A_{\mu}$. We assume that each fluid element consists of quarklike quasiparticles of $N_{f}$ flavors, which admit a classical kinetic description. Following Refs. $[18,23]$ we assume that the single-particle distribution function for particles and antiparticles can be factorized into spin-dependent and spin-independent parts as

$$
f_{s, \mathrm{eq}}^{ \pm}(x, p, s)=f_{\mathrm{eq}}^{ \pm}(x, p) \exp \left(\frac{1}{2} \omega_{\alpha \beta}(x) s^{\alpha \beta}\right),
$$

where $f_{\mathrm{eq}}^{ \pm}(x, p)$ is the stationary solution to the Boltzmann equation, $\omega_{\alpha \beta}$ is the spin polarization tensor (see Sec. III for discussion) and $s^{\alpha \beta}$ is the internal angular momentum [63] for massive spin-1/2 particles defined in terms of spin four vector $s^{\alpha}$ and particle four momentum $p^{\alpha}$ [64]

$$
s^{\alpha \beta}=\frac{1}{m} \epsilon^{\alpha \beta \gamma \delta} p_{\gamma} s_{\delta},
$$

where $m$ is the particle mass.
In the rest of this section, we ignore possible modification of the spin-dependent part of the distribution function due to EM field by assuming that $\omega_{\alpha \beta}$ is small $[18,20,24]$.

\section{A. The stationary solution to the BV equation}

In the presence of EM fields, we employ the stationary solution to the BVequation [65]. The relativistic BV equation in the collisionless limit reads

$$
p^{\mu} \partial_{\mu} f_{i}^{ \pm} \pm q_{i} F^{\mu \nu} p_{\nu} \partial_{\mu}^{p} f_{i}^{ \pm}=0,
$$

where $i=1, \ldots, N_{f}$ is the flavor index, $q_{i}\left(-q_{i}\right)$ is the (anti)particle electric charge for each flavor, and $F_{\mu \nu}=$ $\partial_{\mu} A_{\nu}-\partial_{\nu} A_{\mu}$ is the EM field strength tensor. All quarks have the same baryon number while their electric charges differ. In the global equilibrium, the solution to Eq. (3) reads $[61,65]$

$$
f_{\mathrm{eq}, i}^{ \pm}(x, p)=\exp \left[ \pm \frac{\xi_{\mathrm{B}}}{3}-\beta^{\mu}\left(p_{\mu} \pm q_{i} A_{\mu}\right)\right],
$$

where $\xi_{\mathrm{B}}$ is the ratio of baryon chemical potential $\mu$ over temperature $T, \xi_{\mathrm{B}} \equiv \mu / T$, and $\beta^{\mu}$ is the ratio of fluid flow vector $U^{\mu}$ and temperature, $\beta^{\mu} \equiv U^{\mu} / T$. Plugging the solution (4) into Eq. (3) gives rise to

$$
\frac{1}{2} p^{\mu} p^{\nu} £_{\beta} g_{\mu \nu} \pm q_{i} p^{\mu} £_{\beta} A_{\mu}=0 .
$$

Here $£_{\beta} X$ is the Lie derivative of a tensor with respect to $\beta$ and in particular [66]

$$
\begin{gathered}
£_{\beta} A_{\mu}=\beta^{\nu} \partial_{\nu} A_{\mu}+A_{\nu} \partial_{\mu} \beta^{\nu}, \\
£_{\beta} g_{\mu \nu}=\partial_{\mu} \beta_{\nu}+\partial_{\nu} \beta_{\mu} .
\end{gathered}
$$

Equation (5) is satisfied in the global equilibrium for which we have

$$
£_{\beta} g_{\mu \nu}=0, \quad £_{\beta} A_{\mu}=0 .
$$

We rewrite the latter relation above using Eq. (6) as follows

$$
\begin{aligned}
£_{\beta} A_{\nu} & =\beta^{\mu}\left(\partial_{\mu} A_{\nu}-\partial_{\nu} A_{\mu}\right)+\beta^{\mu} \partial_{\nu} A_{\mu}+A_{\mu} \partial_{\nu} \beta^{\mu}, \\
& =\beta^{\mu} F_{\mu \nu}+\partial_{\nu}(\beta \cdot A)=0 .
\end{aligned}
$$

The Faraday tensor $F_{\mu \nu}$ can be decomposed with respect to the four velocity $U_{\mu}$ in the following way [67]

$$
F_{\mu \nu}=E_{\mu} U_{\nu}-E_{\nu} U_{\mu}+\epsilon_{\mu \nu \alpha \beta} U^{\alpha} B^{\beta},
$$

where the EM four vectors are defined as 


$$
E^{\mu} \equiv F^{\mu \nu} U_{\nu}, \quad B^{\mu} \equiv \frac{1}{2} \epsilon^{\mu \nu \alpha \beta} F_{\nu \alpha} U_{\beta} .
$$

Plugging Eq. (10) into Eq. (9) and expressing $U^{\mu}$ with $\beta^{\mu}$ gives rise to

$$
\frac{E_{\mu}}{T}=\partial_{\mu}(\beta \cdot A)
$$

Integrating Eq. (12) and assuming that $E_{\mu}$ and $T$ are slowly varying at the microscopic scale leads to

$$
\beta \cdot A=\frac{E_{\mu}}{T} \int d x^{\mu},
$$

up to a gauge transformation that should be absorbed into the quark baryon chemical potential $\mu / 3$ [68]. By this virtue, the solution (4) can be rewritten as

$$
f_{\mathrm{eq}, i}^{ \pm}(x, p)=\exp \left( \pm \xi_{i}-\beta^{\mu} p_{\mu}\right)
$$

where

$$
\xi_{i}=\xi_{\mathrm{B}}-q_{i} \frac{E_{\mu}}{T} \int d x^{\mu} .
$$

The distribution function (14) has an important implication. Even if the event-by-event average of the electric field vanishes [51], its fingerprint in the distribution function may survive.

Thus, the spin distribution function Eq. (1), can be written using Eq. (14) in the small polarization limit as

$$
f_{\mathrm{i}, \mathrm{s}, \mathrm{eq}}^{ \pm}(x, p, s)=f_{\mathrm{eq}, i}^{ \pm}(x, p)\left[1+\frac{1}{2} \omega_{\alpha \beta}(x) s^{\alpha \beta}\right] .
$$

\section{B. Baryon and electric charges}

In contrast to Ref. [20], the fluid considered in this work has two different charge currents: a baryon charge current $N^{\alpha}$ and an electric charge current $J^{\alpha}$. In equilibrium we have [24]

$$
N_{\mathrm{eq}}^{\alpha}=\sum_{i}^{N_{f}} \int \mathrm{dPdS} p^{\alpha}\left[f_{\mathrm{i}, \mathrm{s}, \mathrm{eq}}^{+}-f_{\mathrm{i}, \mathrm{s}, \mathrm{eq}}^{-}\right],
$$

where the invariant momentum integration measure $\mathrm{dP}$ and spin integration measure dS is [18]

$$
\mathrm{dP}=\frac{d^{3} p}{(2 \pi)^{3} E_{p}}, \quad \mathrm{~d} \mathrm{~S}=\frac{m}{\pi \mathfrak{S}} \mathrm{d}^{4} s \delta\left(s \cdot s+\mathfrak{g}^{2}\right) \delta(p \cdot s),
$$

and $\mathfrak{s}^{2}=3 / 4$ is the length of the spin vector.
Plugging the distribution function (16) in Eq. (17), and keeping the terms up to first order for small polarization in $\omega_{\mu \nu}$ gives rise to $[1,14]$

$$
N_{\mathrm{eq}}^{\alpha}=n U^{\alpha}=\sum_{i}^{N_{f}} n_{i} U^{\alpha},
$$

where $n_{i}=4 \sinh \left(\xi_{i}\right) n_{(0), i}(T)$ and $n_{(0), i}(T)$ denotes the number density of spinless and neutral massive Boltzmann particles of the form $[1,14]$

$$
n_{(0), i}(T)=\frac{1}{2 \pi^{2}} T^{3} \hat{m}_{i}^{2} K_{2}\left(\hat{m}_{i}\right),
$$

with $\hat{m}_{i} \equiv m_{i} / T$ being the ratio of the $i$ th flavor mass over temperature and $K_{n}\left(\hat{m}_{i}\right)$ is the $n$th modified Bessel function of the second kind.

Baryon charge conservation law has the form

$$
\partial_{\alpha} N_{\text {eq }}^{\alpha}(x)=0,
$$

which is guaranteed by Eq. (3) and it also implies that the baryon charge current is independently conserved for each flavor, namely,

$$
\partial_{\alpha} N_{i, \mathrm{eq}}^{\alpha}(x)=0, \quad \text { with } \quad N_{i, \mathrm{eq}}^{\alpha} \equiv n_{i} U^{\alpha} .
$$

Next we turn to the electric current. First, note that the conservation of electric current is an implication of the inhomogeneous Maxwell equation, namely,

$$
\partial_{\mu} F^{\mu \nu}=J^{\nu}=\rho_{e} U^{\nu}+\Delta^{\nu \rho} J_{\rho},
$$

where $\Delta^{\nu \rho}$ is the spatial projection operator expressed as

$$
\Delta^{\nu \rho}=g^{\nu \rho}-U^{\nu} U^{\rho},
$$

and $\rho_{e}$ is the local electric charge density given by

$$
\rho_{e}=\sum_{i}^{N_{f}} \int \mathrm{dPdS}(p \cdot U) q_{i}\left[f_{\mathrm{i}, \mathrm{s}, \mathrm{eq}}^{+}-f_{\mathrm{i}, \mathrm{s}, \mathrm{eq}}^{-}\right],
$$

which, similar to Eq. (19), gives rise to

$$
\rho_{e}=\sum_{i}^{N_{f}} q_{i} n_{i} .
$$

In what follows, we use Bjorken-expanding resistive MHD in which the symmetries require electric neutrality [55]. The fluid can have a net baryon charge density $n$ with a vanishing electric charge density $\rho_{e}$. Such a setup is simplified but not unrealistic in the later stages of the fireball evolution. Out of equilibrium, the electric current is expressed as 


$$
J^{\mu}=\sigma_{e} E^{\mu},
$$

where $\sigma_{e}$ is the electric conductivity and the above current is dissipative.

\section{Conservation of energy and linear momentum}

In equilibrium, the energy-momentum tensor of the fluid reads

$$
T_{\mathrm{eq}}^{\mu \nu}=\sum_{i}^{N_{f}} \int \mathrm{dPdS} p^{\mu} p^{\nu}\left[f_{\mathrm{i}, \mathrm{s}, \mathrm{eq}}^{+}+f_{\mathrm{i}, \mathrm{seq}}^{-}\right] .
$$

Using Eq. (16) in above equation, we obtain

$$
\partial_{\mu} T_{\mathrm{eq}}^{\mu \nu}=F^{\nu \rho} J_{\rho, \text { eq }} .
$$

However, the above form of the energy-momentum conservation law is implied by the diffeomorphism and gauge invariance [69] and is therefore independent of the underlying microscopic theory. By this virtue, the conservation law of energy and linear momentum has the form

$$
\partial_{\alpha} T_{\text {fluid }}^{\alpha \beta}(x)=F^{\beta \gamma} J_{\gamma},
$$

where for the perfect fluid, the energy-momentum tensor $T_{\mathrm{eq}}^{\alpha \beta}$ is expressed as

$$
T_{\mathrm{eq}}^{\alpha \beta}=(\varepsilon+P) U^{\alpha} U^{\beta}-P g^{\alpha \beta},
$$

with the energy density and pressure having the form

$$
\varepsilon=4 \sum_{i}^{N_{f}} \cosh \left(\xi_{i}\right) \varepsilon_{(0), i}(T),
$$

and

$$
P=4 \sum_{i}^{N_{f}} \cosh \left(\xi_{i}\right) P_{(0), i}(T),
$$

respectively.

Similar to $(20), \varepsilon_{(0), i}(T)$ and $P_{(0), i}(T)$ are the energy density and pressure for spinless and neutral massive Boltzmann particles defined as $[1,14]$

$$
\varepsilon_{(0), i}(T)=\frac{1}{2 \pi^{2}} T^{4} \hat{m}_{i}^{2}\left[\hat{m}_{i} K_{1}\left(\hat{m}_{i}\right)+3 K_{2}\left(\hat{m}_{i}\right)\right],
$$

and

$$
P_{(0), i}(T)=T n_{(0), i}(T)
$$

respectively.

\section{Entropy conservation}

At this stage, we would like to comment on entropy conservation in the presence of background electric fields. The entropy current reads [24]

$$
\begin{aligned}
H^{\mu}= & -\sum_{i}^{N_{f}} \int \mathrm{dPdS} p^{\mu}\left[f_{\mathrm{i}, \mathrm{s}, \mathrm{eq}}^{+}\left(\log f_{\mathrm{i}, \mathrm{s}, \mathrm{eq}}^{+}-1\right)\right. \\
& \left.+f_{\mathrm{i}, \mathrm{s}, \mathrm{eq}}^{-}\left(\log f_{\mathrm{i}, \mathrm{s}, \mathrm{eq}}^{-}-1\right)\right] .
\end{aligned}
$$

Plugging Eq. (1) with Eq. (4) into the above equation, we obtain [24]

$H^{\mu}=P \beta^{\mu}+\beta_{\alpha} T_{\mathrm{eq}}^{\mu \alpha}-\frac{1}{2} \omega_{\alpha \beta} S_{\mathrm{eq}}^{\mu, \alpha \beta}-\sum_{i}^{N_{f}} \xi_{i}(x) N_{i, \mathrm{eq}}^{\mu}$,

where $S_{\mathrm{eq}}^{\mu, \alpha \beta}$ is the spin tensor.

In global equilibrium, where Eq. (8) holds, one has

$$
\begin{aligned}
\partial_{\mu}\left(P \beta^{\mu}+\beta_{\alpha} T_{\mathrm{eq}}^{\mu \alpha}\right) & =\beta_{\alpha} \partial_{\mu} T_{\mathrm{eq}}^{\mu \alpha} \\
& =\beta_{\alpha} F^{\alpha \beta} J_{\beta, \mathrm{eq}}=-\sum_{i}^{N_{f}} q_{i} E_{\mu} N_{i, \mathrm{eq}}^{\mu},
\end{aligned}
$$

wherein Eq. (29) was used. Using the above relation, and Eq. (22) in the divergence of (37) gives rise to

$$
\begin{aligned}
\partial_{\mu} H^{\mu} & =-\sum_{i}^{N_{f}} N_{i, \mathrm{eq}}^{\mu} \partial_{\mu}\left(\xi_{i}(x)+q_{i} E_{\mu}\right), \\
& =-n T \beta^{\mu} \partial_{\mu} \xi_{\mathrm{B}}=-n T £_{\beta} \xi_{\mathrm{B}}=0 .
\end{aligned}
$$

We conclude that the electric field does not induce entropy production in global equilibrium provided the chemical potential is modified according to Eq. (15).

We also point out that the entropy current has the contribution from the polarization. However, since there is no direct coupling between spin and electromagnetic fields in our setup, we have neglected such terms as they were already taken care of in Ref. [24]. We should note that the entropy current analysis does not rely on any approximation, and the Eq. (37) is exact. Equation (39) and the analysis given in Ref. [24] admits that the entropy is conserved in, and only in, equilibrium.

The setup that is worked out in this section is called the strong electric field regime by certain authors [62]. It should be emphasized that in the rest of the current work we consider resistive MHD equations with the electrical conductivity as the only source of dissipation. Out of equilibrium, the entropy production has the form 


$$
\partial_{\mu} H^{\mu}=\frac{\sigma_{e}}{T} E^{2},
$$

where $E \equiv \sqrt{-E^{\mu} E_{\mu}}$.

\section{SPIN POLARIZATION TENSOR AND CONSERVATION OF SPIN ANGULAR MOMENTUM}

\section{A. Spin polarization tensor}

The spin polarization tensor $\omega_{\mu \nu}$ is an antisymmetric rank-two tensor, which can be always expressed as follows

$$
\omega_{\mu \nu}=\kappa_{\mu} U_{\nu}-\kappa_{\nu} U_{\mu}+\epsilon_{\mu \nu \alpha \beta} U^{\alpha} \omega^{\beta},
$$

where $U^{\mu}$ is the fluid four velocity and $\kappa^{\mu}$ and $\omega^{\mu}$ are yet other four vectors [14]. Any part of the $\kappa^{\mu}$ and $\omega^{\mu}$ parallel to $U^{\mu}$ does not contribute to the right-hand side of Eq. (41). Therefore, we assume that $\kappa^{\mu}$ and $\omega^{\mu}$ fulfill the following orthogonality conditions

$$
\kappa \cdot U=0, \quad \omega \cdot U=0 .
$$

Hence $\kappa_{\mu}$ and $\omega_{\mu}$ can be written as

$$
\begin{aligned}
\kappa_{\mu} & =\omega_{\mu \alpha} U^{\alpha} \equiv a_{X} X_{\mu}+a_{Y} Y_{\mu}+a_{Z} Z_{\mu}, \\
\omega_{\mu} & =\frac{1}{2} \epsilon_{\mu \alpha \beta \gamma} \omega^{\alpha \beta} U^{\gamma} \equiv b_{X} X_{\mu}+b_{Y} Y_{\mu}+b_{Z} Z_{\mu} .
\end{aligned}
$$

Here the scalar quantities $a_{X}, a_{Y}, a_{Z}, b_{X}, b_{Y}$, and $b_{Z}$ are called spin polarization coefficients.

A general expression for the spin polarization tensor in terms of $\kappa$ and $\omega$ four vectors has the form [20],

$$
\begin{aligned}
\omega_{\mu \nu}= & a_{X}\left(X_{\mu} U_{\nu}-X_{\nu} U_{\mu}\right)+a_{Y}\left(Y_{\mu} U_{\nu}-Y_{\nu} U_{\mu}\right) \\
& +a_{Z}\left(Z_{\mu} U_{\nu}-Z_{\nu} U_{\mu}\right) \\
& +\epsilon_{\mu \nu \alpha \beta} U^{\alpha}\left(b_{X} X^{\beta}+b_{Y} Y^{\beta}+b_{Z} Z^{\beta}\right),
\end{aligned}
$$

where $X, Y$, and $Z$ together with $U$ form a four-vector basis satisfying the following normalization conditions

$$
\begin{gathered}
U \cdot U=1, \\
X \cdot X=Y \cdot Y=Z \cdot Z=-1, \\
X \cdot U=Y \cdot U=Z \cdot U=0, \\
X \cdot Y=Y \cdot Z=Z \cdot X=0 .
\end{gathered}
$$

\section{B. Conservation of angular momentum}

In the formalism by de Groot, van Leeuwen, and van Weert (GLW) the energy-momentum tensor is symmetric; hence, the angular momentum conservation implies the conservation of the spin tensor and therefore we can write [17]

$$
\partial_{\alpha} S_{\mathrm{GLW}}^{\alpha, \beta \gamma}(x)=0
$$

where in the leading-order spin polarization tensor, the GLW spin tensor is written as $[15,17]$

$$
\begin{aligned}
S_{\mathrm{GLW}}^{\alpha, \beta \gamma}= & \sum_{i}^{N_{f}} \cosh \left(\xi_{i}\right)\left[\mathcal{A}_{1, i}\left(U^{[\beta} \omega^{\gamma] \alpha}+g^{\alpha[\beta} \kappa^{\gamma]}\right)\right. \\
& \left.+\mathcal{A}_{2, i} U^{\alpha} U^{[\beta} \kappa^{\gamma]}+\mathcal{A}_{3, i} U^{\alpha} \omega^{\beta \gamma}\right],
\end{aligned}
$$

with thermodynamic coefficients having forms

$$
\begin{gathered}
\mathcal{A}_{1, i}=-\frac{2}{\hat{m}_{i}^{2}} \frac{\varepsilon_{(0), i}(T)+P_{(0), i}(T)}{T}, \\
\mathcal{A}_{2, i}=2 n_{(0), i}(T)-6 \mathcal{A}_{1, i}, \\
\mathcal{A}_{3, i}=n_{(0), i}-\mathcal{A}_{1, i} .
\end{gathered}
$$

\section{PERFECT-FLUID AND SPIN DYNAMICS}

Based on the discussion given in the previous sections in the following we will study perfect-fluid dynamics in the presence of electromagnetic fields, as given by Eqs. (21) and (30), and, on top of it, we consider spin evolution equations, as determined by Eq. (49).

\section{A. Boost invariant form of conservation laws}

Using Eq. (19), the conservation law for charge Eq. (21) can be cast into the following form

$$
U^{\alpha} \partial_{\alpha} n+n \partial_{\alpha} U^{\alpha} \equiv \frac{d n}{d \tau}+\frac{n}{\tau}=0 .
$$

Projecting Eq. (30) on $U_{\beta}$ and then using Eq. (31), we also obtain

$$
\begin{aligned}
U^{\alpha} \partial_{\alpha} \varepsilon+(\varepsilon+P) \partial_{\alpha} U^{\alpha} & =\sigma_{e} E^{2}, \\
\frac{d \varepsilon}{d \tau}+\frac{\varepsilon+P}{\tau} & =\sigma_{e} E^{2} .
\end{aligned}
$$

Using Eqs. (44) and (50) in Eq. (49), and contracting the resulting tensor equation with $U_{\beta} X_{\gamma}, U_{\beta} Y_{\gamma}, U_{\beta} Z_{\gamma}, Y_{\beta} Z_{\gamma}$, $X_{\beta} Z_{\gamma}$, and $X_{\beta} Y_{\gamma}$ we obtain the following evolution equations for the spin polarization coefficients $\boldsymbol{a}=\left(a_{X}, a_{Y}, a_{Z}\right.$, $\left.b_{X}, b_{Y}, b_{Z}\right)[20]$,

$\operatorname{diag}(\mathcal{L}, \mathcal{L}, \mathcal{L}, \mathcal{P}, \mathcal{P}, \mathcal{P}) \dot{\boldsymbol{a}}=\operatorname{diag}\left(\mathcal{Q}, \mathcal{Q}, \mathcal{Q}_{1}, \mathcal{R}, \mathcal{R}, \mathcal{R}_{1}\right) \boldsymbol{a}$ 
respectively, where $(\ldots) \equiv U \cdot \partial=\partial_{\tau}$ and,

$$
\begin{aligned}
\mathcal{L}(\tau) & =\mathcal{A}_{1, i}, \\
\mathcal{P}(\tau) & =\mathcal{A}_{3, i}, \\
\mathcal{Q}(\tau) & =-\left[\dot{\mathcal{L}}+\frac{3 \mathcal{L}}{2 \tau}\right], \\
\mathcal{Q}_{1}(\tau) & =\left[\mathcal{Q}+\frac{\mathcal{L}}{2 \tau}\right], \\
\mathcal{R}(\tau) & =-\left[\dot{\mathcal{P}}+\frac{1}{\tau}\left(\mathcal{P}-\frac{\mathcal{L}}{2}\right)\right], \\
\mathcal{R}_{1}(\tau) & =\left[\mathcal{R}-\frac{\mathcal{L}}{2 \tau}\right]
\end{aligned}
$$

c.f. Eq. (44).

\section{B. Evolution of the EM fields}

To obtain the evolution of EM fields, one needs to simultaneously solve the Maxwell equations and the conservation laws for energy and linear momentum. In general, this is not an easy task. However, here we adopt the nonrotating or maximally boost invariant solution presented in Ref. [55], which is given by

$$
B^{\mu}=B_{0} \frac{\tau_{0}}{\tau} Y^{\mu}, \quad E^{\mu}=\ell E_{0} \frac{\tau_{0}}{\tau} \mathrm{e}^{-\sigma_{e}\left(\tau-\tau_{0}\right)} Y^{\mu},
$$

where $B_{0}$ and $E_{0}$ are the initial values of the magnetic and electric field at the initial proper time $\tau_{0}$, respectively, whereas the parameter $\ell \equiv \frac{B \cdot E}{B E}= \pm 1$, corresponds to the parallel and antiparallel field configurations. The solution to the resistive MHD equations mentioned above is found as follows. The translational symmetries of the Bjorken flow do not permit the magnetic field to exist in the longitudinal $(z)$ direction [57], as well as boost invariance requires both $E_{z}$ and electric charge density $\rho_{e}$ to vanish. Thus, both electric and magnetic fields are constrained to exist only in the transverse, i.e., $x-y$, plane. To preserve the Bjorken flow, the acceleration due to the Poynting vector must vanish, which implies that the electric and magnetic fields are either parallel or antiparallel to each other. The angle between the fields is either $0(\ell=1)$ or $\pi(\ell=-1)$, which remains fixed during the evolution and is a part of the initial conditions. If one assumes that the direction of the fields is also boost invariant, the solution (58) is found from the Maxwell's equations. We should also emphasize that the magnetic field exists in the system, however it does not play any direct role in our setup. Plugging Eq. (58) into Eq. (15), gives rise to

$$
\xi_{i}=\xi_{\mathrm{B}}-\ell q_{i} R_{\mathrm{RMS}} \frac{E_{0}}{T}\left(\frac{\tau_{0}}{\tau}\right) \mathrm{e}^{-\sigma_{e}\left(\tau-\tau_{0}\right)},
$$

where $R_{\mathrm{RMS}}$ is the nucleon root-mean-square charge radius. The solution (58) implies that

$$
\rho_{e}=0 \text {. }
$$

We note here that in our setup the neutrality is not automatically satisfied at the initial time. To see this, consider $\rho_{e}$ at the initial time for $N_{f}=3$. The neutrality puts a constraint on the initial number densities

$$
2 n_{u}\left(\tau_{0}\right)-n_{d}\left(\tau_{0}\right)-n_{s}\left(\tau_{0}\right)=0
$$

As it turns out, such a constraint cannot be satisfied with the physical parameters introduced here, and there is no reason to believe that it should be satisfied by the fluid in realistic situations. On the other hand, as the fluid starts to evolve the local electric charge density relaxes to negligible values in a fraction of a Fermi, and, to a very good approximation, neutrality is reached.

\section{NUMERICAL RESULTS}

In this section we present numerical results for the hydrodynamic variables obtained by solving Eqs. (54) and (55) in the presence of external electric field. These results are then used to solve Eqs. (56) for the spin polarization coefficients. The calculations are performed for two choices of collision energies, i.e., $\sqrt{s_{\mathrm{NN}}}=27 \mathrm{GeV}$ and $\sqrt{s_{\mathrm{NN}}}=200 \mathrm{GeV}$. The initial values for the temperature and baryon chemical potential for each collision energy read as [70]

$T_{0}=300 \mathrm{MeV}, \quad \mu_{0}=300 \mathrm{MeV}$ for $\sqrt{s_{\mathrm{NN}}}=27 \mathrm{GeV}$, $T_{0}=600 \mathrm{MeV}, \quad \mu_{0}=50 \mathrm{MeV}$ for $\sqrt{s_{\mathrm{NN}}}=200 \mathrm{GeV}$.

We use the constituent quark masses at $\Lambda$ 's mass scale for number of quark flavors $N_{f}=3$ [71]

$$
m_{u}=m_{d}=0.382 \mathrm{GeV}, \quad m_{s}=0.537 \mathrm{GeV} .
$$

The initial proper time for both energies is chosen to be $\tau_{0}=1 \mathrm{fm}$, and we adopt $R_{\mathrm{RMS}}=4.3 \mathrm{fm}$ from Ref. [72]. The electric conductivity to second order approximation in $\mu / T$ is given by [73]

$$
\sigma_{e}(T, \mu)=0.37 Q_{e} T\left[1+0.15\left(\frac{\mu}{T}\right)^{2}\right],
$$

where $Q_{e}=(2 / 3) e^{2}$ is the sum over flavors of the quark electric charges squared. To employ different values for the initial electric field $E_{0}$, we introduce the following parameter 


$$
\alpha \equiv \ell \frac{e E_{0}}{m_{\pi}^{2}}
$$

where we choose $e E_{0} / m_{\pi}^{2}=1$ for $\sqrt{s_{\mathrm{NN}}}=200 \mathrm{GeV}$ and scale it down linearly with $\sqrt{s_{\mathrm{NN}}}$ for $\sqrt{s_{\mathrm{NN}}}=27 \mathrm{GeV}$ [51]. As a result, $\alpha$ is our only free parameter for each chosen collision energy. To understand the dynamics of $T$ and $\mu$, it is suitable to rewrite Eq. (55) in the following form

$$
\tau \frac{d \varepsilon}{d \tau}=-w+\sigma_{e} \tau E^{2},
$$

where, $w=\varepsilon+P$ is the enthalpy density.

As the above form suggests, the evolution of energy density is determined by the competition between the expansion term, $\sim w$, and Joule heating $(\mathrm{JH})$ term. The electric field plays two opposite roles. First, it produces entropy and therefore increases the temperature compared to the case without an electric field. To realize this, assume an uncharged conformal fluid with the equation of state, $\varepsilon=3 P=3 f_{P} T^{4}$, with $f_{P}$ being a pure number [4]. Then Eq. (65) is transformed to

$$
\frac{d \log T}{d \log \tau}=-\frac{1}{3}+\frac{\sigma_{e} \tau}{12 f_{P}}\left(\frac{E}{T^{2}}\right)^{2}
$$

The second term on the right hand side increases the temperature and the heating effect gets enhanced by increasing the values of $E / T^{2}$ and $\sigma_{e} \tau$. However, according to Eq. (63), the factor $\sigma_{e} \tau$ is a small number during the hydrodynamic evolution, and increasing it suppresses the electric field, see Eq. (58). On the other hand, if the initial electric field is sufficiently large, the JH term may still dominate over expansion in early times. In such a case, a reheating effect is possible [55]. Nevertheless, the electric field in our setup modifies the dynamics of fluid not only through the JH term but also through the equation of state. Consequently, the analytical investigation presented in Ref. [55] for the reheating conditions is not applicable. Still, some important observations can be made using numerical inspections, such as studying the $\tau$ derivatives of $T$ and $\mu$ at the initial time for various values of $\alpha$ : see Fig. 1, which shows that the reheating observed in Refs. $[53,55]$ is not possible in our setup. For an early-time reheating to occur at a particular value of $\alpha \neq 0$, the initial derivative of $T$ must be positive. It means that if the initial derivative of $T$ is larger than the derivative at $\alpha=0$, then the $\mathrm{JH}$ effect comes into play making the fluid hotter. Inversely, if the initial derivative of $T$ is smaller than the derivative at $\alpha=0$, then the electric field is making the fluid cooler. This electric cooling effect occurs because the electric field makes the fluid elements heavier, which in consequence increases their enthalpy density, as can be seen in Fig. 2. For small $\mu / T$, this effect can be seen using a Taylor expansion of $w$ in $\mu / T$ which reads
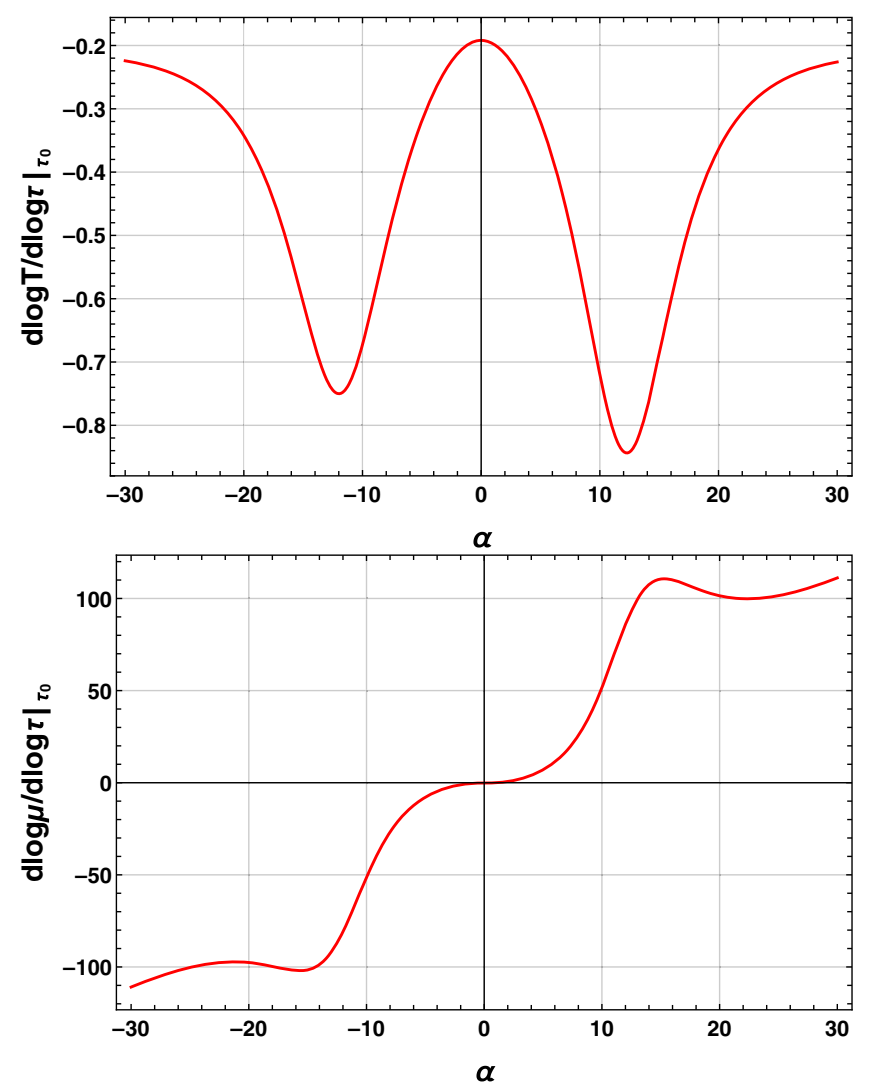

FIG. 1. The quantity $(d \log T) /(d \log \tau)$ (upper panel) and $(d \log \mu) /(d \log \tau)$ (lower panel) as a function of $\alpha$ at $\tau=\tau_{0}$ for the $\sqrt{s_{\mathrm{NN}}}=200 \mathrm{GeV}$.

$$
\begin{aligned}
w_{i}= & 4 \cosh \left(q_{i} \ell \frac{E R_{\mathrm{RMS}}}{T}\right)\left(1-\tanh \left(q_{i} \ell \frac{E R_{\mathrm{RMS}}}{T}\right) \frac{\mu}{3 T}\right. \\
& \left.+\frac{1}{2}\left(\frac{\mu}{3 T}\right)^{2}+\mathcal{O} \frac{\mu 3}{3 T}\right) w_{(0), i}(T),
\end{aligned}
$$

where $w_{(0), i}=\varepsilon_{(0), i}+P_{(0), i}$. Here, the overall factor $\cosh \left(q_{i} \ell \frac{E R_{\mathrm{RMS}}}{T}\right)$ is larger than one. The second term in the parenthesis is a quantity with an absolute value smaller than one with the sign given by $\operatorname{sgn}\left(q_{i} \ell\right)$. Therefore, for $q_{i} \ell<0$ the electric field enhances the enthalpy density slightly more than in the opposite case, see Fig. 2. Since there are more negatively charged quarks than positively charged ones in $N_{f}=3$ case, for $\ell<0$, the enthalpy density is larger which can be seen in lower collision energies where $\mu / T$ is of order unity. However, this difference is negligible in small $\mu / T$ regime. Consequently, the temperature dynamics is similar for both negative and positive values of $\alpha$, as can be seen in the lower panel of Fig. 3. This larger enthalpy density may result a lower temperature for $\alpha<0$. Nevertheless, this is not pronounced unless $\alpha$ is extremely large. For moderate values of $\alpha$ shown in the plots, the asymmetry of Fig. 2 is not rendered into asymmetry of temperatures for positive and negative $\alpha$, as is seen in the upper panel of Fig. 3 . 


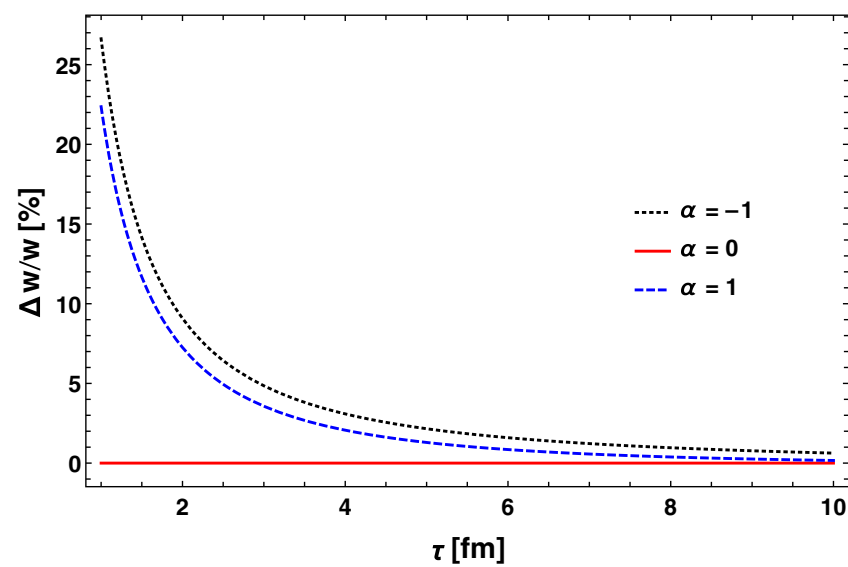

FIG. 2. Percentage of enthalpy density enhancement with respect to $\alpha=0$ case: $\Delta w / w \equiv\left[(\varepsilon+P)_{\alpha \neq 0}-(\varepsilon+P)_{\alpha=0}\right] /$ $(\varepsilon+P)_{\alpha=0}$. The figure is for the $\sqrt{s_{\mathrm{NN}}}=27 \mathrm{GeV}$ case.

By further inspection of the initial derivative of $T$, we can understand the relation between the value of $\alpha$ and electric cooling effect. As it turns out, there is an interval around $|\alpha|=0$, for which increasing $|\alpha|$ enhances the electric
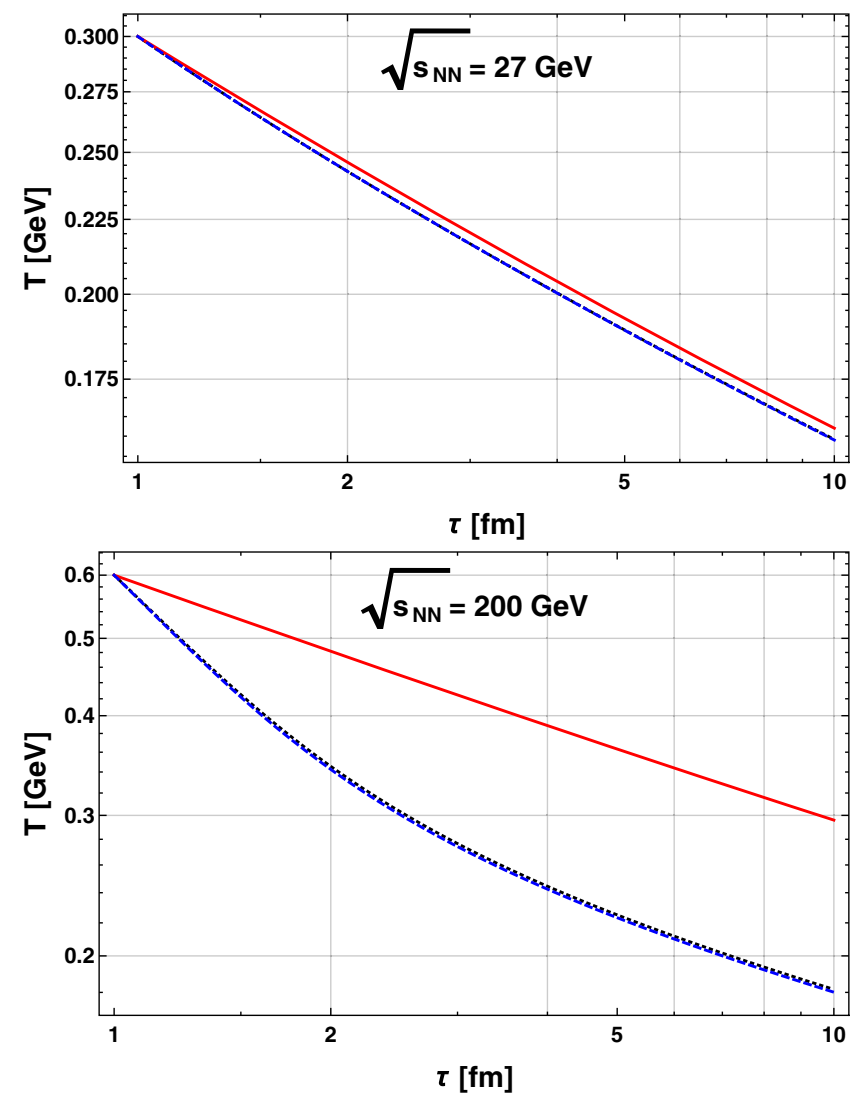

FIG. 3. Temperature profile with initial temperature $T_{0}=$ $300 \mathrm{MeV}$ for $\sqrt{s_{\mathrm{NN}}}=27 \mathrm{GeV}$ (upper panel) and $T_{0}=$ $600 \mathrm{MeV}$ for $\sqrt{s_{\mathrm{NN}}}=200 \mathrm{GeV}$ (lower panel). Dotted black line is for $\alpha=-8$, red line is for $\alpha=0$, and dashed blue line is for $\alpha=8$. cooling effect (see the peak around $\alpha=0$ in the upper panel of Fig. 1). When $|\alpha|$ reaches a threshold, i.e., the two minima in the upper panel of Fig. 1, this behavior is turned opposite, and the temperature starts rising with $|\alpha|$. This is because the $\mathrm{JH}$ term is getting strong enough to partially counterbalance the electric cooling effect. As Fig. 3 suggests, the electric field effects in the dynamics of temperature is less pronounced for larger values of $\mu / T$, since $\mu$ dominates over $E$. We should emphasize that, from a phenomenological perspective, the occurrence of early time reheating is unlikely. If existed, such a reheating should have been already observed, for instance, via electromagnetic probes.

As the lower panel of Fig. 1 indicates, the dynamics of chemical potential is much more sensitive to the electric field than the dynamics of temperature. This is confirmed by Fig. 4. In the small $\mu / T$ regime (the lower panel in Fig. 4), the late-time absolute value of $\mu$ is always larger for $\alpha \neq 0$ case, and $\mu$ has the same sign as $\alpha$. On the other hand, for $\mu / T$ of order unity (the upper panel in Fig. 4), $\mu$ dominates over the electric field and the change of sign requires very large electric fields. Consequently, if $\alpha$ is not too large, $\mu$ is enhanced (suppressed) for positive (negative) values of $\alpha$. Moreover, as the results on $\mu / T$ suggest, see
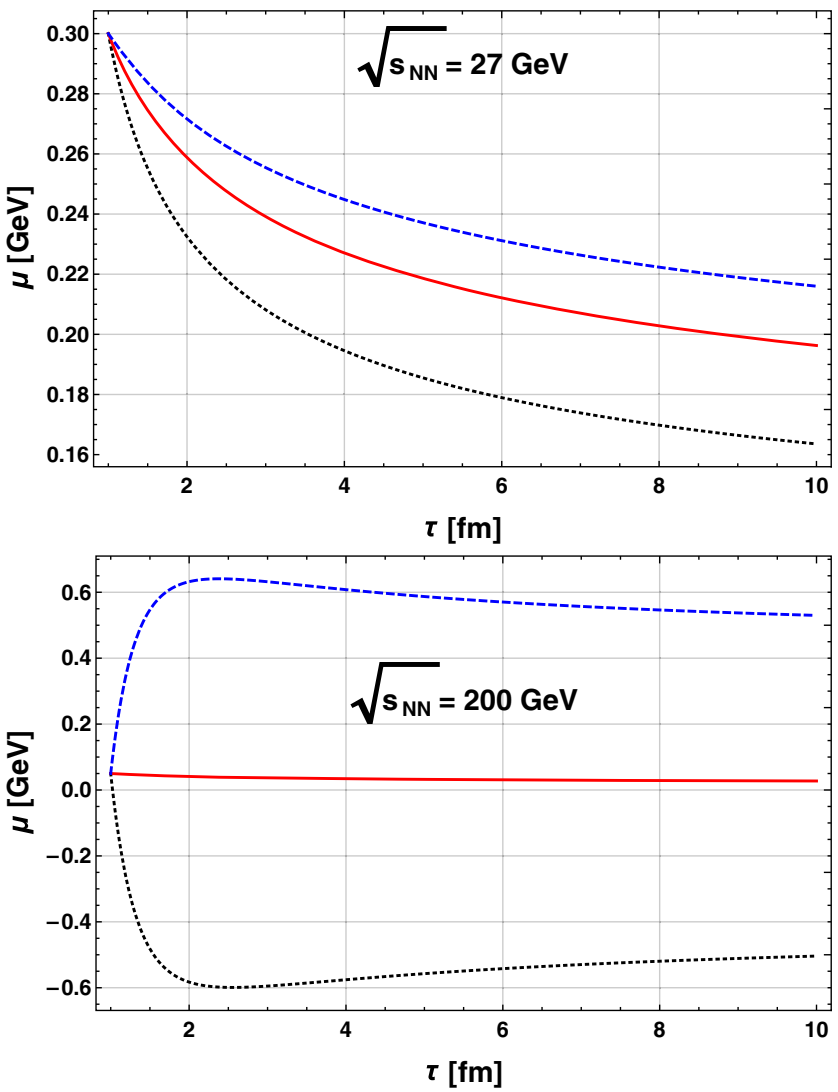

FIG. 4. Baryon chemical potential evolution with initial baryon chemical potential $\mu_{0}=300 \mathrm{MeV}$ for $\sqrt{s_{\mathrm{NN}}}=27 \mathrm{GeV}$ (upper panel) and $\mu_{0}=50 \mathrm{MeV}$ for $\sqrt{s_{\mathrm{NN}}}=200 \mathrm{GeV}$ (lower panel). 

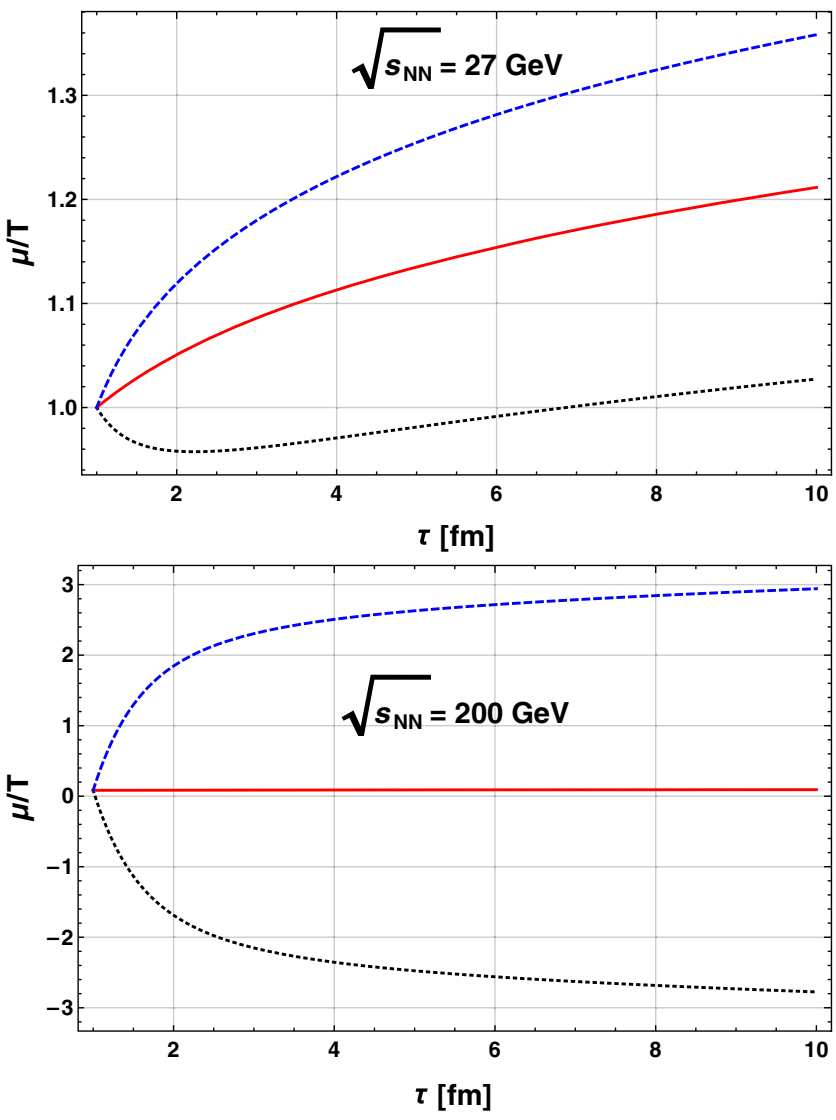

FIG. 5. Ratio of baryon chemical potential over temperature for $\sqrt{s_{\mathrm{NN}}}=27 \mathrm{GeV}$ (upper panel) and $\sqrt{s_{\mathrm{NN}}}=200 \mathrm{GeV}$ (lower panel).

Fig. 5, the electric field modifies the trajectory which the fireball passes through the QCD phase diagram.

Finally, we find that the behavior of the spin polarization components, see Fig. (6), is qualitatively similar to the case of pure Bjorken-expanding perfect-fluid background without an electric field [20]; however, introducing the external electric field in the perfect-fluid background solely interestingly enhances the dynamics of spin polarization coefficients. We also observe that the enhancement of the spin polarization coefficients depends on the ratio $\mu_{0} / T_{0}$. Slope rises drastically for the small value of $\mu_{0} / T_{0}$, i.e., in the higher beam energy case. Hence, our results suggest that the external electric field in the background may play an important role in the polarization dynamics in heavy-ion collisions. In this work we present only the behavior of $b_{Z}$ component; however, all other spin polarization components exhibit similar qualitative features.

The spin polarization coefficients studied in this work is directly related to the spin polarization of the hyperon's emitted from the system at the freeze-out through the formula for the mean spin polarization per particle $\left\langle\pi_{\mu}(p)\right\rangle[17,20]$
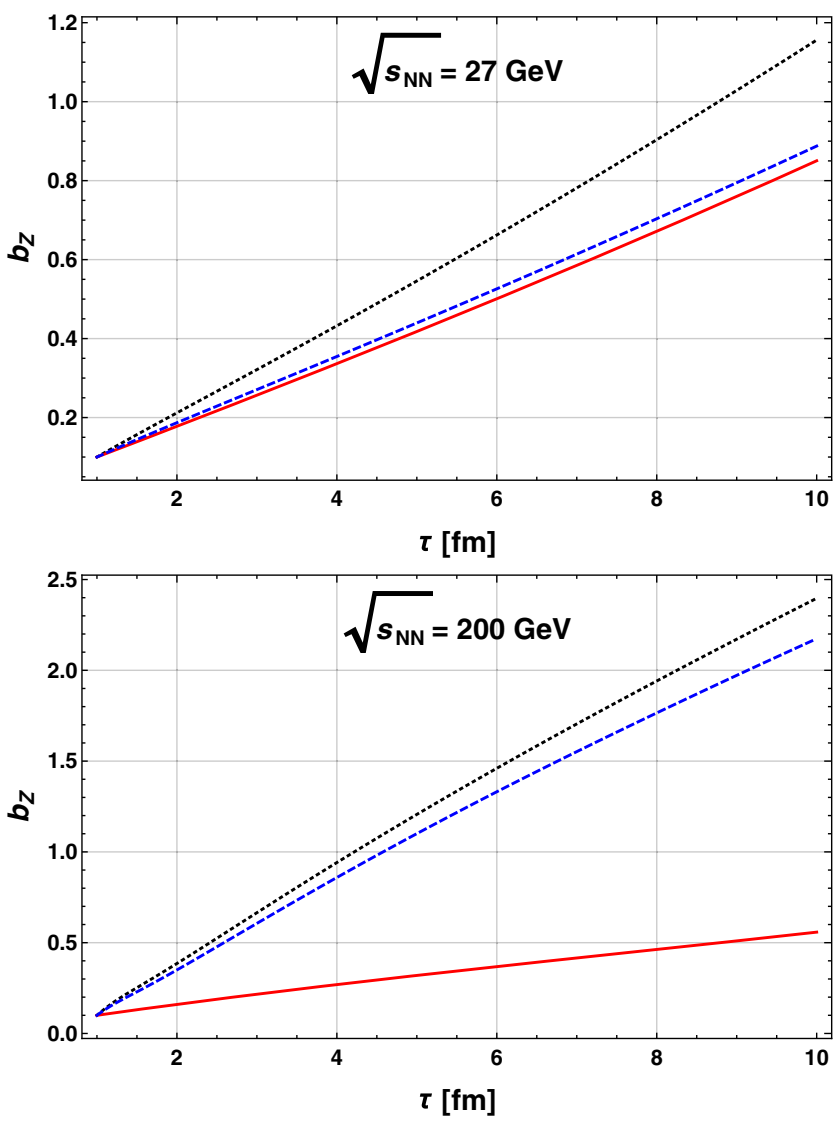

FIG. 6. Spin polarization coefficient $b_{Z}$ profile for $\sqrt{s_{\mathrm{NN}}}=$ $27 \mathrm{GeV}$ (upper panel) and $\sqrt{s_{\mathrm{NN}}}=200 \mathrm{GeV}$ (lower panel) with initial value $b_{Z}^{0}=0.1$. The modification of the $b_{Z}$ evolution slope due to electric field is much more pronounced when $\mu_{0} / T_{0}$ is small as can be seen in the lower panel.

$$
\left\langle\pi_{\mu}\right\rangle=\frac{E_{p} \frac{d \Pi_{\mu}(p)}{d^{3} p}}{E_{p} \frac{d \mathcal{N}(p)}{d^{3} p}} .
$$

The above equation is the ratio of the invariant momentum distribution of the total Pauli-Lubański vector and the momentum density of particles and antiparticles expressed as

$$
E_{p} \frac{d \Pi_{\mu}(p)}{d^{3} p}=\frac{\cosh \left(\xi_{\mathrm{B}}\right)}{(2 \pi)^{3} m} \int \Delta \Sigma_{\lambda} p^{\lambda} e^{-\beta \cdot p} \tilde{\omega}_{\beta \mu} p^{\beta}
$$

and

$$
E_{p} \frac{d \mathcal{N}(p)}{d^{3} p}=\frac{4 \cosh \left(\xi_{\mathrm{B}}\right)}{(2 \pi)^{3}} \int \Delta \Sigma_{\lambda} p^{\lambda} e^{-\beta \cdot p}
$$

respectively, where $\tilde{\omega}^{\mu \nu}=(1 / 2) \epsilon^{\mu \nu \alpha \beta} \omega_{\alpha \beta}$ is the dual polarization tensor [20] and $\Delta \Sigma_{\lambda}$ is the infinitesimal element of the freeze-out hypersurface.

We find that due to the enhancement of the spin polarization coefficients, the dynamics of the average spin 
polarization of $\Lambda$ hyperons, Eq. (68), also gets enhanced and exhibit similar qualitative behavior as compared to the case without electric field in the perfect-fluid Bjorkenexpanding background studied in Ref. [20].

\section{SUMMARY AND CONCLUSIONS}

In the present work, we have studied the spin polarization dynamics in a Bjorken-expanding perfect-fluid resistive MHD background. In equilibrium, we used the stationary solution to the Boltzmann-Vlasov equation to find the modification of the baryon density, energy density, pressure, and energy-momentum tensor in the presence of an external electric field. We have assumed that the fluid is described by the BV solution for a medium composed of noninteracting quarklike quasiparticles. The latter results were used in the MHD equations. From the Maxwell equations and the Bjorken symmetry the evolution of EM fields is readily determined. The symmetry implies that the electric and magnetic field in the local rest frame must be either parallel or antiparallel and oriented in the transverse directions. In such a solution, there is no extra source of fluid acceleration due to the Poynting term.

The MHD equations in our case are reduced to the energy-momentum conservation and the baryon number conservation. We have solved these two equations numerically to find the evolution of temperature and baryon chemical potential and found that the electric field plays a twofold role in this regard. On the one hand, the electric field produces entropy through the $\mathrm{JH}$ term and therefore increases the temperature. On the other hand, it makes the fluid enthalpy density larger, which results in a faster decrease of the temperature. For the collision energies that we have studied, this electric cooling effect is always dominant over the JH term. Therefore our results show a faster decay of temperature than in the purely hydrodynamic case. The dynamics of baryon chemical potential depends on the initial value of $\mu / T$. For small values of $\mu_{0} / T_{0}$, the parallel and antiparallel EM field cases are almost symmetric, and the baryon chemical potential changes sign with the sign of $\alpha$, while its absolute value is always larger than in the purely hydrodynamic case $(\alpha=0)$. On the other hand, for larger values of $\mu_{0} / T_{0}$, the evolution of baryon chemical potential is different for parallel and antiparallel configurations. The antiparallel field cases (i.e., for $\alpha<0$ ) have lower temperature and the baryon chemical potential decreases faster than the purely hydrodynamic case $(\alpha=0)$, while the baryon chemical potential of the parallel fields (i.e., for $\alpha>0$ ) are larger than in the purely hydrodynamic case.

The dynamics of temperature and baryon chemical potential is used to solve the spin conservation law. The resulting spin polarization dynamics has qualitatively similar behavior as in the case of perfect-fluid Bjorkenexpanding background without electric field, but we observe that the dynamics of the spin polarization coefficients is enhanced due to the presence of electric fields in the background. It was found that for small values of $\mu_{0} / T_{0}$, the slope is steeper, and behavior gets enhanced significantly. Therefore, it suggests that the electric field may play a significant role in the polarization dynamics of $\Lambda$ hyperons if it is sufficiently large.

In the present work, the simplest available solution to the resistive MHD was employed, in which the symmetries of Bjorken flow are fully preserved. This implies that the qualitative behavior of the spin polarization remains unchanged. In a more realistic setup, one may consider the modification of the flow and breakdown of the symmetries induced by the EM fields, which may change the dynamics of the spin polarization-we leave these problems for future investigations. Other possible extension of the present work is to include the coupling between the EM fields and spin degrees of freedom at the microscopic level. Investigations along these lines are ongoing and will be reported elsewhere.

\section{ACKNOWLEDGMENTS}

We thank W. Florkowski, I. Karpenko, D. Rischke, N. Sadooghi, and A. Tabatabaee for fruitful discussions. This research was supported in part by the Polish National Science Centre Grants No. 2016/23/B/ST2/00717 and No. 2018/30/E/ST2/00432.
[1] W. Florkowski, Phenomenology of Ultra-Relativistic Heavy-Ion Collisions (World Scientific, Singapore, 2010), https://cds.cern.ch/record/1321594.

[2] A. Jaiswal and V. Roy, Relativistic hydrodynamics in heavy-ion collisions: General aspects and recent developments, Adv. High Energy Phys. 2016, 9623034 (2016).
[3] P. Romatschke and U. Romatschke, Relativistic Fluid Dynamics In and Out of Equilibrium, Cambridge Monographs on Mathematical Physics Vol. 5 (Cambridge University Press, Cambridge, England, 2019).

[4] W. Florkowski, M. P. Heller, and M. Spalinski, New theories of relativistic hydrodynamics in the LHC era, Rep. Prog. Phys. 81, 046001 (2018). 
[5] B. Schenke, The smallest fluid on earth, arXiv:2102.11189.

[6] C. Gale, S. Jeon, and B. Schenke, Hydrodynamic modeling of heavy-ion collisions, Int. J. Mod. Phys. A 28, 1340011 (2013).

[7] M. P. Heller and M. Spalinski, Hydrodynamics Beyond the Gradient Expansion: Resurgence and Resummation, Phys. Rev. Lett. 115, 072501 (2015).

[8] M. Shokri and F. Taghinavaz, Conformal Bjorken flow in the general frame and its attractor: Similarities and discrepancies with the Müller-Israel-Stewart formalism, Phys. Rev. D 102, 036022 (2020).

[9] L. Adamczyk et al. (STAR Collaboration), Global $\Lambda$ hyperon polarization in nuclear collisions: Evidence for the most vortical fluid, Nature (London) 548, 62 (2017).

[10] J. Adam et al. (STAR Collaboration), Global polarization of $\Lambda$ hyperons in $\mathrm{Au}+\mathrm{Au}$ collisions at $\sqrt{s_{N N}}=200 \mathrm{GeV}$, Phys. Rev. C 98, 014910 (2018).

[11] J. Adam et al. (STAR Collaboration), Polarization of $\Lambda(\bar{\Lambda})$ Hyperons Along the Beam Direction in $\mathrm{Au}+\mathrm{Au}$ Collisions at $\sqrt{s_{N N}}=200 \mathrm{GeV}$, Phys. Rev. Lett. 123, 132301 (2019).

[12] S. Acharya et al. (ALICE Collaboration), Global polarization of $\Lambda \bar{\Lambda}$ hyperons in $\mathrm{Pb}-\mathrm{Pb}$ collisions at $\sqrt{s_{N N}}=2.76$ and 5.02 TeV, Phys. Rev. C 101, 044611 (2020).

[13] F. Kornas et al. (HADES Collaboration), Lambda Polarization in $\mathrm{Au}+\mathrm{Au}$ collisions at $\sqrt{s_{N N}}=2.4 \mathrm{GeV}$ measured with HADES, in Proceedings at the Strange Quark Matter, Bari, Italy (2019).

[14] W. Florkowski, B. Friman, A. Jaiswal, and E. Speranza, Relativistic fluid dynamics with spin, Phys. Rev. C 97, 041901 (2018).

[15] W. Florkowski, B. Friman, A. Jaiswal, R. Ryblewski, and E. Speranza, Spin-dependent distribution functions for relativistic hydrodynamics of spin-1/2 particles, Phys. Rev. D 97, 116017 (2018).

[16] F. Becattini, W. Florkowski, and E. Speranza, Spin tensor and its role in non-equilibrium thermodynamics, Phys. Lett. B 789, 419 (2019).

[17] W. Florkowski, A. Kumar, and R. Ryblewski, Thermodynamic versus kinetic approach to polarization-vorticity coupling, Phys. Rev. C 98, 044906 (2018).

[18] W. Florkowski, R. Ryblewski, and A. Kumar, Relativistic hydrodynamics for spin-polarized fluids, Prog. Part. Nucl. Phys. 108, 103709 (2019).

[19] W. Florkowski, A. Kumar, R. Ryblewski, and A. Mazeliauskas, Longitudinal spin polarization in a thermal model, Phys. Rev. C 100, 054907 (2019).

[20] W. Florkowski, A. Kumar, R. Ryblewski, and R. Singh, Spin polarization evolution in a boost invariant hydrodynamical background, Phys. Rev. C 99, 044910 (2019).

[21] R. Singh, G. Sophys, and R. Ryblewski, Spin polarization dynamics in the Gubser-expanding background, Phys. Rev. D 103, 074024 (2021).

[22] L. Tinti and W. Florkowski, Particle polarization, spin tensor and the Wigner distribution in relativistic systems, arXiv: 2007.04029.

[23] S. Bhadury, W. Florkowski, A. Jaiswal, A. Kumar, and R. Ryblewski, Relativistic dissipative spin dynamics in the relaxation time approximation, Phys. Lett. B 814, 136096 (2021).
[24] S. Bhadury, W. Florkowski, A. Jaiswal, A. Kumar, and R. Ryblewski, Dissipative Spin Dynamics in Relativistic Matter, Phys. Rev. D 103, 014030 (2021).

[25] S. Shi, C. Gale, and S. Jeon, From chiral kinetic theory to relativistic viscous spin hydrodynamics, Phys. Rev. C 103, 044906 (2021).

[26] W. Florkowski and R. Ryblewski, On the interpretation of $\Lambda$ spin polarization measurements, arXiv:2102.02890.

[27] A. D. Gallegos, U. Gürsoy, and A. Yarom, Hydrodynamics of spin currents, arXiv:2101.04759.

[28] S. Y. F. Liu and Y. Yin, Spin polarization induced by the hydrodynamic gradients, arXiv:2103.09200.

[29] B. Fu, S. Y. F. Liu, L. Pang, H. Song, and Y. Yin, Shearinduced spin polarization in heavy-ion collisions, arXiv: 2103.10403.

[30] F. Becattini and L. Tinti, The Ideal relativistic rotating gas as a perfect fluid with spin, Ann. Phys. (Amsterdam) 325, 1566 (2010).

[31] F. Becattini, V. Chandra, L. Del Zanna, and E. Grossi, Relativistic distribution function for particles with spin at local thermodynamical equilibrium, Ann. Phys. (Amsterdam) 338, 32 (2013).

[32] F. Becattini, L. Csernai, and D. J. Wang, $\Lambda$ polarization in peripheral heavy ion collisions, Phys. Rev. C 88, 034905 (2013); Erratum, Phys. Rev. C 93, 069901 (2016).

[33] M. I. Baznat, K. K. Gudima, A. S. Sorin, and O. V. Teryaev, Femto-vortex sheets and hyperon polarization in heavy-ion collisions, Phys. Rev. C 93, 031902 (2016).

[34] F. Becattini, I. Karpenko, M. Lisa, I. Upsal, and S. Voloshin, Global hyperon polarization at local thermodynamic equilibrium with vorticity, magnetic field and feed-down, Phys. Rev. C 95, 054902 (2017).

[35] I. Karpenko and F. Becattini, Study of $\Lambda$ polarization in relativistic nuclear collisions at $\sqrt{s_{\mathrm{NN}}}=7.7200 \mathrm{GeV}$, Eur. Phys. J. C 77, 213 (2017).

[36] Y. Xie, D. Wang, and L. P. Csernai, Global $\Lambda$ polarization in high energy collisions, Phys. Rev. C 95, 031901 (2017).

[37] Y. Sun and C. M. Ko, $\Lambda$ hyperon polarization in relativistic heavy ion collisions from a chiral kinetic approach, Phys. Rev. C 96, 024906 (2017).

[38] H. Li, L.-G. Pang, Q. Wang, and X.-L. Xia, Global $\Lambda$ polarization in heavy-ion collisions from a transport model, Phys. Rev. C 96, 054908 (2017).

[39] F. Becattini and I. Karpenko, Collective Longitudinal Polarization in Relativistic Heavy-Ion Collisions at Very High Energy, Phys. Rev. Lett. 120, 012302 (2018).

[40] D.-X. Wei, W.-T. Deng, and X.-G. Huang, Thermal vorticity and spin polarization in heavy-ion collisions, Phys. Rev. C 99, 014905 (2019).

[41] X.-L. Xia, H. Li, Z.-B. Tang, and Q. Wang, Probing vorticity structure in heavy-ion collisions by local $\Lambda$ polarization, Phys. Rev. C 98, 024905 (2018).

[42] Y. Sun and C. M. Ko, Azimuthal angle dependence of the longitudinal spin polarization in relativistic heavy ion collisions, Phys. Rev. C 99, 011903 (2019).

[43] J.-H. Gao and Z.-T. Liang, Relativistic quantum kinetic theory for massive fermions and spin effects, Phys. Rev. D 100, 056021 (2019). 
[44] Y. B. Ivanov, V. Toneev, and A. Soldatov, Vorticity and particle polarization in relativistic heavy-ion collisions, Phys. At. Nucl. 83, 179 (2020).

[45] J. I. Kapusta, E. Rrapaj, and S. Rudaz, Hyperon polarization in relativistic heavy ion collisions and axial U(1) symmetry breaking at high temperature, Phys. Rev. C 101, 031901 (2020).

[46] J.-H. Gao, Z.-T. Liang, Q. Wang, and X.-N. Wang, Global polarization effect and spin-orbit coupling in strong interaction, arXiv:2009.04803.

[47] X.-G. Deng, X.-G. Huang, Y.-G. Ma, and S. Zhang, Vorticity in low-energy heavy-ion collisions, Phys. Rev. C 101, 064908 (2020).

[48] W. M. Serenone, J. a. G. P. Barbon, D. D. Chinellato, M. A. Lisa, C. Shen, J. Takahashi, and G. Torrieri, $\Lambda$ polarization from thermalized jet energy, arXiv:2102.11919.

[49] D. Rindori, L. Tinti, F. Becattini, and D. Rischke, Relativistic quantum fluid with boost invariance, arXiv:2102 .09016.

[50] D. E. Kharzeev, L. D. McLerran, and H. J. Warringa, The effects of topological charge change in heavy ion collisions: 'Event by event $\mathrm{P}$ and $C P$ violation', Nucl. Phys. A803, 227 (2008).

[51] X.-G. Huang, Electromagnetic fields and anomalous transports in heavy-ion collisions-A pedagogical review, Rep. Prog. Phys. 79, 076302 (2016).

[52] L. Oliva, P. Moreau, V. Voronyuk, and E. Bratkovskaya, Influence of electromagnetic fields in proton-nucleus collisions at relativistic energy, Phys. Rev. C 101, 014917 (2020).

[53] V. Roy, S. Pu, L. Rezzolla, and D. Rischke, Analytic Bjorken flow in one-dimensional relativistic magnetohydrodynamics, Phys. Lett. B 750, 45 (2015).

[54] S. Pu, V. Roy, L. Rezzolla, and D. H. Rischke, Bjorken flow in one-dimensional relativistic magnetohydrodynamics with magnetization, Phys. Rev. D 93, 074022 (2016).

[55] M. Shokri and N. Sadooghi, Novel self-similar rotating solutions of nonideal transverse magnetohydrodynamics, Phys. Rev. D 96, 116008 (2017).

[56] W. Florkowski, A. Kumar, and R. Ryblewski, Vortex-like solutions and internal structures of covariant ideal magnetohydrodynamics, Eur. Phys. J. A 54, 184 (2018).

[57] M. Shokri and N. Sadooghi, Evolution of magnetic fields from the $3+1$ dimensional self-similar and Gubser flows in ideal relativistic magnetohydrodynamics, J. High Energy Phys. 11 (2018) 181.

[58] M. Shokri, Generalization of Bantilan-Ishi-Romatschke flow to Magnetohydrodynamics, J. High Energy Phys. 01 (2020) 011.

[59] G. Inghirami, L. Del Zanna, A. Beraudo, M. H. Moghaddam, F. Becattini, and M. Bleicher, Numerical magneto- hydrodynamics for relativistic nuclear collisions, Eur. Phys. J. C 76, 659 (2016).

[60] G. Inghirami, M. Mace, Y. Hirono, L. Del Zanna, D. E. Kharzeev, and M. Bleicher, Magnetic fields in heavy ion collisions: Flow and charge transport, Eur. Phys. J. C 80, 293 (2020).

[61] N. Weickgenannt, X.-L. Sheng, E. Speranza, Q. Wang, and D. H. Rischke, Kinetic theory for massive spin-1/2 particles from the Wigner-function formalism, Phys. Rev. D 100, 056018 (2019).

[62] J. Hernandez and P. Kovtun, Relativistic magnetohydrodynamics, J. High Energy Phys. 05 (2017) 001.

[63] M. Mathisson, Neue mechanik materieller systemes, Acta Phys. Pol. 6, 163 (1937), https://inspirehep.net/literature/ 48323.

[64] C. Itzykson and J. B. Zuber, Quantum Field Theory, International Series In Pure and Applied Physics (McGraw-Hill, New York, 1980), http://dx.doi.org/10 $.1063 / 1.2916419$.

[65] S. De Groot, Relativistic Kinetic Theory. Principles and Applications (North-Holland Publishing Company, Amsterdam, 1980), Vol. 1, https://inspirehep.net/literature/ 162065.

[66] L. Parker and D. Toms, Quantum Field Theory in Curved Spacetime: Quantized Fields and Gravity, Cambridge Monographs on Mathematical Physics (Cambridge University Press, Cambridge, England, 2009).

[67] J. D. Bekenstein and E. Oron, New conservation laws in general-relativistic magnetohydrodynamics, Phys. Rev. D 18, 1809 (1978).

[68] P. Kovtun, Thermodynamics of polarized relativistic matter, J. High Energy Phys. 07 (2016) 028.

[69] K. Jensen, M. Kaminski, P. Kovtun, R. Meyer, A. Ritz, and A. Yarom, Towards Hydrodynamics Without an Entropy Current, Phys. Rev. Lett. 109, 101601 (2012).

[70] I. A. Karpenko, P. Huovinen, H. Petersen, and M. Bleicher, Estimation of the shear viscosity at finite net-baryon density from $A+A$ collision data at $\sqrt{s_{\mathrm{NN}}}=7.7-200 \mathrm{GeV}$, Phys. Rev. C 91, 064901 (2015).

[71] V. Borka Jovanovic, S. R. Ignjatovic, D. Borka, and P. Jovanovic, Constituent quark masses obtained from hadron masses with contributions of Fermi-Breit and GlozmanRiska hyperfine interactions, Phys. Rev. D 82, 117501 (2010).

[72] S. S. Gubser, S. S. Pufu, and A. Yarom, Entropy production in collisions of gravitational shock waves and of heavy ions, Phys. Rev. D 78, 066014 (2008).

[73] G. Aarts and A. Nikolaev, Electrical conductivity of the quark-gluon plasma: Perspective from lattice QCD, Eur. Phys. J. A 57, 118 (2021). 